University of Nebraska - Lincoln

DigitalCommons@University of Nebraska - Lincoln

Timothy J. Gay Publications

Research Papers in Physics and Astronomy

February 1989

Elastic angular differential cross sections for quasi-oneelectron collision systems at intermediate energies: $\left(\mathrm{Na}^{+}, \mathrm{Li}^{+}\right)+\mathrm{H}$ and $\left(\mathrm{Mg}^{+}\right.$, $\left.\mathrm{Be}^{+}\right)+\mathrm{He}$

\author{
J. L. Peacher \\ University of Missouri-Rolla, Rolla, Missouri \\ E. Redd \\ University of Missouri-Rolla, Rolla, Missouri \\ D. G. Seely \\ University of Missouri-Rolla, Rolla, Missouri \\ Timothy J. Gay \\ University of Nebraska - Lincoln, tgay1@unl.edu \\ D. M. Blakenship \\ University of Missouri-Rolla, Rolla, Missouri \\ See next page for additional authors \\ Follow this and additional works at: https://digitalcommons.unl.edu/physicsgay \\ Part of the Physics Commons
}

Peacher, J. L.; Redd, E.; Seely, D. G.; Gay, Timothy J. ; Blakenship, D. M.; and Park, J. T., "Elastic angular differential cross sections for quasi-oneelectron collision systems at intermediate energies: $\left(\mathrm{Na}^{+}, \mathrm{Li}^{+}\right)+\mathrm{H}$ and $\left(\mathrm{Mg}^{+}, \mathrm{Be}^{+}\right)+\mathrm{He}^{\prime \prime}(1989)$. Timothy J. Gay Publications. 16.

https://digitalcommons.unl.edu/physicsgay/16

This Article is brought to you for free and open access by the Research Papers in Physics and Astronomy at DigitalCommons@University of Nebraska - Lincoln. It has been accepted for inclusion in Timothy J. Gay Publications by an authorized administrator of DigitalCommons@University of Nebraska - Lincoln. 


\section{Authors}

J. L. Peacher, E. Redd, D. G. Seely, Timothy J. Gay, D. M. Blakenship, and J. T. Park

This article is available at DigitalCommons@University of Nebraska - Lincoln: https://digitalcommons.unl.edu/ 
Phys. Rev. A 39, 1760 - 1766 (1989)

[Issue 4 - February 1989]

\section{Elastic angular differential cross sections for quasi-one- electron collision systems at intermediate energies: $\left(\mathrm{Na}^{+}\right.$, $\left.\mathrm{Li}^{+}\right)+\mathrm{H}$ and $\left(\mathrm{Mg}^{+}, \mathrm{Be}^{+}\right)+\mathrm{He}$}

J. L. Peacher, E. Redd, D. G. Seely, T. J. Gay, D. M. Blankenship, and J. T. Park Physics Department and Laboratory of Atomic and Molecular Research, University of Missouri-Rolla, Rolla, Missouri 65401

Measurements of elastic angular differential cross sections have been carried out for four quasi-one-electron collision systems at intermediate energies. Data are presented for $\mathrm{Na}^{+}+\mathrm{H}$ collisions at laboratory energies of $35.94,51.75,63.89$, and $143.75 \mathrm{keV}$, for $\mathrm{Li}^{+}+\mathrm{H}$ collisions at energies of 19.44 and $43.75 \mathrm{keV}$, for $\mathrm{Mg}^{+}+\mathrm{He}$ collisions at energies of $30,66.7$, and $150 \mathrm{keV}$, and for $\mathrm{Be}^{+}+\mathrm{He}$ collisions at an energy of $56.25 \mathrm{keV}$. The highest energy in each case corresponds to a projectile velocity of $(1 / 2$ a.u. Born and Eikonal calculations, in which we model the projectile ion as a heavy structureless ion of charge +1 e, are also presented. Our model calculations are in fair agreement with the experimental data over the range of measured scattering angles.

(C)1989 The American Physical Society

URL: http://link.aps.org/abstract/PRA/v39/p1760

DOI: 10.1103/PhysRevA.39.1760

PACS: $34.40 .+n$ 


\title{
Elastic angular differential cross sections for quasi-one-electron collision systems at intermediate energies: $\left(\mathrm{Na}^{+}, \mathrm{Li}^{+}\right)+\mathrm{H}$ and $\left(\mathrm{Mg}^{+}, \mathrm{Be}^{+}\right)+\mathrm{He}$
}

\author{
J. L. Peacher, E. Redd, ${ }^{*}$ D. G. Seely, T. J. Gay, D. M. Blankenship, ${ }^{\dagger}$ and J. T. Park \\ Physics Department and Laboratory of Atomic and Molecular Research, University of Missouri-Rolla, Rolla, Missouri 65401
}

(Received 31 May 1988; revised manuscript received 25 August 1988)

\begin{abstract}
Measurements of elastic angular differential cross sections have been carried out for four quasione-electron collision systems at intermediate energies. Data are presented for $\mathrm{Na}^{+}+\mathrm{H}$ collisions at laboratory energies of $35.94,51.75,63.89$, and $143.75 \mathrm{keV}$, for $\mathrm{Li}^{+}+\mathrm{H}$ collisions at energies of 19.44 and $43.75 \mathrm{keV}$, for $\mathrm{Mg}^{+}+\mathrm{He}$ collisions at energies of $30,66.7$, and $150 \mathrm{keV}$, and for $\mathrm{Be}^{+}+\mathrm{He}$ collisions at an energy of $56.25 \mathrm{keV}$. The highest energy in each case corresponds to a projectile velocity of $\frac{1}{2}$ a.u. Born and Eikonal calculations, in which we model the projectile ion as a heavy structureless ion of charge $+1 e$, are also presented. Our model calculations are in fair agreement with the experimental data over the range of measured scattering angles.
\end{abstract}

\section{INTRODUCTION}

Elastic scattering of an ion by a gas target is conceptually the simplest ion-atom collision process because the projectile ion is scattered by the target atom with no changes in the internal states of either collision partner. However, the determination of the angular differential cross section for this process presents a challenge both theoretically and experimentally.

Theoretically, the treatment of the elastic scattering process is difficult at intermediate energies (where the projectile velocity is comparable to the velocity of a valence electron) because the other possible inelastic channels, especially ionization and charge transfer, could significantly couple to the elastic channel. This effect was very pronounced in comparisons of our elastic protonhelium data with theory. ${ }^{1}$ Our first Born and Glauber (or Eikonal) calculations ${ }^{1}$ for this system using a static potential, which did not contain any coupling to the other scattering channels, overestimated the elastic angular differential cross section (ADCS) significantly. At 50 and $100 \mathrm{keV}$ the Glauber calculation was about a factor of 10 higher than the data at large scattering angles. The Born calculation, as expected, did even worse over the whole energy range. It is well known that the Glauber approximation ensures that probability flux is conserved, i.e., the Glauber approximation satisfies unitarity in contrast to the Born approximation. ${ }^{2}$ If the cross sections for the inelastic channels are small compared to the elastic cross section, they will have little effect on the elastic cross section because they would represent only a small probability flux. In this case perturbative approximations can be used to calculate the cross sections. On the other hand, if the cross sections for the inelastic channels are comparable to the elastic cross section, they will have a large effect on the elastic cross section because they would represent a significant amount of flux. In the former case the elastic flux is nearly equal to the incident flux. In the latter case the incident flux will divide comparably between the elastic and inelastic channels. Kobayashi and Ishihara ${ }^{3}$ carried out a full Glauber calculation for the elastic proton-helium collision. The full Glauber approximation implicitly takes into account the influence of oth- er channels on the elastic scattering. This led to some improvement in the theoretical results. Their results agreed fairly well with the experimental data at $25 \mathrm{keV}$. At 50 and $100 \mathrm{keV}$ their results were larger than the experimental data by about a factor of 2 and 4, respectively, at all angles. The reason is unclear but may indicate that the theoretical calculation does not handle all inelastic channels equally well. Recently Potvliege, Furtado, and Joachain ${ }^{4}$ have analyzed the problem within the framework of the second-order optical-potential method. This approach attempts to explicitly take into account the influence of the excitation, ionization, and charge transfer channels on the elastic scattering. The calculated results using this method were only slightly improved over the full Glauber results of Kobayashi and Ishihara. Their results varied from being about a factor of 4 too large at the lower measured angles to being about a factor of 2.5 too large at the larger measured angles. Both theoretical groups were unable to account for the discrepancy between the theoretical and experimental results for elastic proton-helium scattering. On the other hand, the full Glauber results are in fairly good accord with the experimental data for elastic proton-hydrogen scattering. ${ }^{5}$

Experimentally, the necessity to distinguish elastically scattered ions from both the inelastically scattered ions and the unscattered ions requires high resolution in both energy loss and scattering angle. The high-energy resolution of our apparatus permits the elastically scattered ions to be separated from the inelastically scattered ions at all angles. However, it is not possible to distinguish elastic events from the unscattered zero energy-loss ions near zero scattering angle. High angular resolution and extremely narrow beam collimation are necessary in order to measure the very small scattering angles which dominate in this intermediate energy range.

This paper reports both our experimental and model calculation results for the elastic ADCS for various quasi-one-electron collision systems in the intermediate energy range. Quasi-one-electron (QOE) collision systems involve an outer valence electron and either two closed-shell cores or one closed shell and a bare nucleus. The relatively simple structure of such systems makes them an obvious choice for study in order to learn about 
fundamental collision processes. Direct excitation in QOE collision systems was recently reviewed. ${ }^{6}$ The QOE collision systems considered in this paper are $\mathrm{Mg}^{+}$and $\mathrm{Be}^{+}$colliding with $\mathrm{He}$ and $\mathrm{Na}^{+}$and $\mathrm{Li}^{+}$colliding with H. These collision systems represent part of a series of investigations on QOE collision systems. We have investigated the ADCS for the excitation of a core electron in helium in $\mathrm{Be}^{+}+\mathrm{He}$ and $\mathrm{Mg}^{+}+\mathrm{He}$ collisions ${ }^{7}$ as well as the ADCS for the excitation of the valence electron in $\mathrm{Mg}^{+}+\mathrm{He}$ and $\mathrm{Na}^{+}+\mathrm{H}$ collisions. ${ }^{8}$ In addition, we have recently investigated the charge transfer ADCS for the $\mathrm{Be}^{+}+\mathrm{He}$ and $\mathrm{Mg}^{+}+\mathrm{He}$ collision systems. ${ }^{9}$ This paper is an extension of our investigations of QOE systems to elastic ADCS's. Also included in this paper are our model calculations of the elastic ADCS using Born and Eikonal approximations with the static potential of the target.

The measurements reported here are the only elastic ADCS measurements for these QOE collision systems. The only other elastic ADCS measurements in this intermediate energy range are our proton-helium ${ }^{1}$ and proton-hydrogen ${ }^{5}$ measurements. Measurements prior to our angular measurements in this intermediate energy range were of total $\mathrm{ADCS}^{6}{ }^{6}$ and thus did not result in a genuine elastic ADCS because the inelastic scattering was not adequately separated from the elastic scattering. The quasi-one-electron collision systems studied here are an extension of our work on the one-electron protonhydrogen scattering system.

\section{EXPERIMENTAL METHOD}

A description of the University of Missouri-Rolla (UMR) differential ion energy-loss spectrometer and the general method employed have been presented previously in detail. ${ }^{1,5,7-15}$ The relevant details are summarized here. The apparatus is a linear dc accelerator-decelerator system which consists of three major sections. These are the source preparation section, the collision section, and the analysis section.

In the source preparation section, ions are produced by electron bombardment or surface ionization in a Colutron G-2 ion gun. The ions are initially accelerated to 2$\mathrm{keV}$ energy and pass through a Colutron Wien velocity filter. The resulting mass-selected ion beam is then accelerated to the desired collision energy, focused, and steered into the collision region.

In the collision section, the incident ions collide with target gas atoms. In this experiment the scattering chamber was one of two variable-angle differentially pumped collision chambers, one for hydrogen and one for helium. The hydrogen target scattering chamber ${ }^{14}$ consists of two coaxial Joule-heated tungsten tubes. Molecular hydrogen is admitted between the tubes where it dissociates. Dissociation fractions are typically greater than $95 \%$ in these experiments. The resulting gas effuses into the scattering volume where it encounters the incident ion beam, which has been collimated along the common axis of the tungsten tubes. The helium-target scattering chamber ${ }^{9}$ has very short differential pumping regions and thin-walled, small-diameter gas-containment apertures.
Because of these features the escaping gas has a negligible effect on the gas density in the scattering chamber. The pressure in both scattering chambers is controlled by a microprocessor-based pressure controller. ${ }^{16}$

In the analysis section, the scattered ions pass through a switching magnet into a decelerator. The switching magnet separates the ions of different charge. The scattered ions as well as the unscattered ions are diverted into the entrance of the decelerator. These ions are decelerated to the pass energy of an electrostatic analyzer. Most of the $\mathrm{H}$ target results were obtained using a $127^{\circ}$ cylindrical analyzer ${ }^{17}$ while all of the $\mathrm{He}$ target results were obtained using a $135^{\circ}$ hemispherical analyzer. ${ }^{13}$ An energy-loss spectrum is obtained by keeping the pass energy constant while varying the energy of the incident ions. This is done by increasing the accelerator voltage by a specific amount. When the extra energy obtained by the ion due to the increased accelerator voltage is equal to the energy lost in a collision, that ion passes through the decelerator and analyzer and is detected. Energy-loss spectra as a function of scattering angle are obtained by pivoting the accelerator part of the apparatus about the center of the scattering chamber.

The experimental apparatus has an angular resolution of $120 \mu \mathrm{rad}$ (lab frame) and a typical energy resolution of one part in $10^{5}$. The spectra associated with the data presented here were taken with an energy resolution of approximately $1 \mathrm{eV}$, determined by the energy width of the source. These characteristics permit an unambiguous identification of the elastically scattered ions. Of course the elastically scattered ions cannot be distinguished from the unscattered zero-energy-loss ions near $0^{\circ}$ scattering angle. Therefore the elastic ADCS's reported here do not start at $0^{\circ}$ scattering angle, but at some nonzero scattering angle which is determined by the angular spread of the incident ion beam. The general method of correcting for the angular spread of the incident ion beam and the finite size of the detector is given in Ref. 11. A description of how the elastic data were handled in order to use our general method of correction is given in Ref. 1 .

\section{THEORETICAL METHOD}

The simplest theoretical model that one can use for elastic scattering in these collisions is to treat the incident ion as structureless with a charge $+1 e$, i.e., the incident ion is treated as a "heavy proton." One would expect this to be a useful model only for very-small-angle (largeimpact-parameter) collisions for which the ion core does not significantly overlap the target core. Although this is not the case for the collisions considered here, it will be demonstrated later that this simple model does remarkably well.

First Born and Eikonal calculations ${ }^{18}$ for this "heavyproton" model were carried out using the static HartreeFock potential field of the target atoms. Cox and Bonham ${ }^{19}$ expressed the static potential as a sum of screened Coulomb potentials with adjustable range and strength parameters. They determined the parameters by fitting this analytical form of the potential to the potential obtained from the Hartree-Fock wave functions calculated 
by Clementi and Roetti. ${ }^{20}$ The theoretical calculations were simplified considerably by using this analytical form of the static potential. The Born approximation is straightforward in this case because the Born scattering amplitude is obtained in analytical form for a sum of screened Coulomb potentials. Likewise, the calculation for the Eikonal scattering amplitude is simplified although the integral over the impact parameter must still be carried out numerically.

\section{RESULTS AND DISCUSSION}

The data we have obtained for elastic collisions are presented in Tables I-IV. The data are also shown in Figs. 1-3 along with the results of our "heavy-proton" model calculations. The energies correspond to a range of relative collision velocities from $\frac{1}{4}-\frac{1}{2}$ a.u. The shape of the displayed experimental data is similar for all of the quasi-one-electron collision systems considered here; they are peaked in the forward direction and fall from 3 to 4 orders of magnitude in the observed angular range.

The errors indicated in the figures and tables were taken to be the quadrative sum of the statistical and overall random measurement errors. The statistical errors were obtained by averaging together the final results of a number of angular runs for a given energy. The random measurement errors were determined by considering the uncertainties in the various quantities that are measured in order to obtain an ADCS. They amounted to $10 \%$ for a hydrogen target and $7.4 \%$ for a helium target. ${ }^{15}$ Only one run was taken for $\mathrm{Li}^{+}+\mathrm{H}$ at $19.44 \mathrm{keV}$. Thus for

TABLE I. Experimentally determined elastic angular differential cross sections for sodium-ion-hydrogen scattering in the centerof-mass frame for a sodium ion with different laboratory energies.

\begin{tabular}{|c|c|c|c|c|}
\hline $\begin{array}{c}\theta_{\text {c.m. }} \\
(\mathrm{mrad})\end{array}$ & $\begin{array}{c}35.94 \mathrm{keV} \\
d \sigma / d \Omega \\
\left(\mathrm{cm}^{2} / \mathrm{sr}\right)\end{array}$ & $\begin{array}{c}51.75 \mathrm{keV} \\
d \sigma / d \Omega \\
\left(\mathrm{cm}^{2} / \mathrm{sr}\right) \\
\end{array}$ & $\begin{array}{c}63.89 \mathrm{keV} \\
d \sigma / d \Omega \\
\left(\mathrm{cm}^{2} / \mathrm{sr}\right)\end{array}$ & $\begin{array}{c}143.75 \mathrm{keV} \\
d \sigma / d \Omega \\
\left(\mathrm{cm}^{2} / \mathrm{sr}\right)\end{array}$ \\
\hline 6.0 & & & & $(4.2 \pm 0.5) \times 10^{-13}$ \\
\hline 7.5 & & & & $(1.5 \pm 0.2) \times 10^{-13}$ \\
\hline 8.0 & $(6.9 \pm 1.7) \times 10^{-13}$ & & & \\
\hline 9.0 & & $(3.1 \pm 0.9) \times 10^{-13}$ & $(2.5 \pm 0.3) \times 10^{-13}$ & $(5.6 \pm 0.9) \times 10^{-14}$ \\
\hline 10.0 & $(3.8 \pm 1.0) \times 10^{-13}$ & $(2.2 \pm 0.6) \times 10^{-13}$ & $(1.9 \pm 0.2) \times 10^{-13}$ & \\
\hline 11.0 & & & & $(2.5 \pm 0.4) \times 10^{-14}$ \\
\hline 12.0 & $(3.0 \pm 0.9) \times 10^{-13}$ & $(1.1 \pm 0.4) \times 10^{-13}$ & $(1.0 \pm 0.1) \times 10^{-13}$ & $(1.5 \pm 0.2) \times 10^{-14}$ \\
\hline 14.0 & & $(5.8 \pm 2.2) \times 10^{-14}$ & $(5.5 \pm 0.6) \times 10^{-14}$ & $(8.6 \pm 1.3) \times 10^{-15}$ \\
\hline 15.0 & $(1.3 \pm 0.4) \times 10^{-13}$ & & & \\
\hline 16.0 & & $(3.4 \pm 1.4) \times 10^{-14}$ & $(2.9 \pm 0.5) \times 10^{-14}$ & $(5.8 \pm 0.9) \times 10^{-15}$ \\
\hline 17.0 & $(9.1 \pm 2.4) \times 10^{-14}$ & & & \\
\hline 18.0 & & & & $(4.2 \pm 0.9) \times 10^{-15}$ \\
\hline 20.0 & $(5.2 \pm 1.4) \times 10^{-14}$ & $(1.3 \pm 0.6) \times 10^{-14}$ & & $(3.2 \pm 0.8) \times 10^{-15}$ \\
\hline 22.0 & & & & $(2.5 \pm 0.8) \times 10^{-15}$ \\
\hline 23.0 & $(2.9 \pm 0.8) \times 10^{-14}$ & & & \\
\hline 24.0 & & $(4.4 \pm 1.9) \times 10^{-15}$ & $(4.4 \pm 0.5) \times 10^{-15}$ & \\
\hline 25.0 & & & & $(2.0 \pm 0.8) \times 10^{-15}$ \\
\hline 27.0 & $(1.5 \pm 0.4) \times 10^{-14}$ & & & \\
\hline 28.0 & & & & $(1.1 \pm 0.3) \times 10^{-15}$ \\
\hline 29.6 & & $(1.7 \pm 0.7) \times 10^{-15}$ & & \\
\hline 30.0 & & & & $(6.3 \pm 1.8) \times 10^{-16}$ \\
\hline 31.0 & $(9.2 \pm 2.4) \times 10^{-15}$ & & & \\
\hline 32.0 & & & $(8.0 \pm 1.5) \times 10^{-16}$ & \\
\hline 35.0 & & $(1.4 \pm 0.2) \times 10^{-15}$ & & \\
\hline 39.0 & $(2.7 \pm 0.8) \times 10^{-15}$ & & & \\
\hline 40.0 & & $(7.1 \pm 1.1) \times 10^{-16}$ & $(2.7 \pm 0.4) \times 10^{-16}$ & \\
\hline 42.5 & & $(6.2 \pm 1.7) \times 10^{-16}$ & & \\
\hline 46.5 & & $(3.6 \pm 0.4) \times 10^{-16}$ & & \\
\hline 47.0 & $(1.1 \pm 0.3) \times 10^{-15}$ & & & \\
\hline 48.0 & & & $(1.1 \pm 0.1) \times 10^{-16}$ & \\
\hline 50.5 & & $(2.2 \pm 0.6) \times 10^{-16}$ & & \\
\hline 54.5 & & $(1.7 \pm 0.4) \times 10^{-16}$ & & \\
\hline 55.0 & $(6.3 \pm 1.7) \times 10^{-16}$ & & & \\
\hline 56.0 & & & $(6.3 \pm 0.8) \times 10^{-17}$ & \\
\hline 59.7 & & $(1.2 \pm 0.5) \times 10^{-16}$ & & \\
\hline 63.0 & $(3.5 \pm 1.0) \times 10^{-16}$ & & & \\
\hline 63.7 & & & $(4.5 \pm 1.2) \times 10^{-17}$ & \\
\hline 71.0 & $(2.7 \pm 0.9) \times 10^{-16}$ & & & \\
\hline 78.0 & $(1.4 \pm 0.5) \times 10^{-16}$ & & & \\
\hline
\end{tabular}


TABLE II. Experimentally determined elastic angular differential cross sections for lithium-ion-hydrogen scattering in the center-of-mass frame for a lithium ion with different laboratory energies.

\begin{tabular}{|c|c|c|}
\hline $\begin{array}{c}\theta_{\text {c.m }} \\
(\mathrm{mrad})\end{array}$ & $\begin{array}{c}19.44 \mathrm{keV} \\
d \sigma / d \Omega \\
\left(\mathrm{cm}^{2} / \mathrm{sr}\right)\end{array}$ & $\begin{array}{c}43.75 \mathrm{keV} \\
d \sigma / d \Omega \\
\left(\mathrm{cm}^{2} / \mathrm{sr}\right)\end{array}$ \\
\hline 2.5 & $(8.0 \pm 1.3) \times 10^{-12}$ & \\
\hline 3.0 & $(5.2 \pm 1.3) \times 10^{-12}$ & $(1.7 \pm 0.3) \times 10^{-12}$ \\
\hline 3.5 & $(2.3 \pm 0.4) \times 10^{-12}$ & \\
\hline 4.0 & & $(5.7 \pm 1.3) \times 10^{-13}$ \\
\hline 4.3 & $(1.7 \pm 0.3) \times 10^{-12}$ & \\
\hline 5.0 & & $(2.6 \pm 0.5) \times 10^{-13}$ \\
\hline 5.1 & $(1.1 \pm 0.1) \times 10^{-12}$ & \\
\hline 6.5 & $(4.4 \pm 0.5) \times 10^{-13}$ & $(7.9 \pm 1.0) \times 10^{-14}$ \\
\hline 7.8 & $(1.8 \pm 0.2) \times 10^{-13}$ & \\
\hline 8.0 & & $(2.9 \pm 0.4) \times 10^{-14}$ \\
\hline 10.0 & & $(9.4 \pm 1.8) \times 10^{-15}$ \\
\hline 10.5 & $(7.0 \pm 1.6) \times 10^{-14}$ & \\
\hline 11.0 & & $(5.2 \pm 1.1) \times 10^{-15}$ \\
\hline 12.0 & & $(3.4 \pm 0.6) \times 10^{-15}$ \\
\hline 13.1 & $(1.3 \pm 0.4) \times 10^{-14}$ & \\
\hline 13.5 & & $(2.9 \pm 1.0) \times 10^{-15}$ \\
\hline 15.3 & & $(1.3 \pm 0.4) \times 10^{-15}$ \\
\hline 15.8 & $(9.6 \pm 2.3) \times 10^{-15}$ & \\
\hline 18.5 & $(2.6 \pm 1.1) \times 10^{-15}$ & \\
\hline
\end{tabular}

this system the statistical error associated with each data point was obtained by considering the percent deviation in the total number of counts. This was determined by counting over four or more gate times to obtain the average count rate and the statistical deviation associated with the average value. ${ }^{15}$

One of the purposes of studying QOE collision systems is to look for commonality between the various systems. It is of interest to see if the results for different projectile ions follow the same simple scaling that we observed for different hydrogen-isotope projectiles. ${ }^{21}$ The differential cross section was expressed as

$$
d \sigma / d \Omega=\mu^{2} F(v, q),
$$

where $\mu$ is the reduced mass, $v$ is the relative collision velocity, and $q$ is the momentum transfer. The $F$ is some function of $v$ and $q$. For a very small scattering angle $\theta$, $q=\mu v \theta$. We found that the various hydrogen-isotope collision systems at the same $v$ yielded the same curve if $\left(1 / \mu^{2}\right)(d \sigma / d \Omega)$ were plotted versus $\mu v \theta$. In that experiment the projectile was a structureless ion, which corresponds to the theoretical model that we have used here. However, all of the projectile ions in the experiments reported here have attached electrons, which complicates the theoretical description of the collision process. The reduced masses are nearly equal for the $\mathrm{Li}^{+}+\mathrm{H}$ and $\mathrm{Na}^{+}+\mathrm{H}$ collision systems. Therefore the ADCS for these two collision systems should be nearly identical for the same relative collision velocity if the simple scaling is

TABLE III. Experimentally determined elastic angular differential cross sections for magnesiumion-helium scattering in the center-of-mass frame for a magnesium ion with different laboratory energies.

\begin{tabular}{|c|c|c|c|}
\hline $\begin{array}{c}\theta_{\mathrm{c} . \mathrm{m} .} \\
(\mathrm{mrad})\end{array}$ & $\begin{array}{l}30 \mathrm{keV} \\
d \sigma / d \Omega \\
\left(\mathrm{cm}^{2} / \mathrm{sr}\right)\end{array}$ & $\begin{array}{c}66.7 \mathrm{keV} \\
d \sigma / d \Omega \\
\left(\mathrm{cm}^{2} / \mathrm{sr}\right)\end{array}$ & $\begin{array}{c}150 \mathrm{keV} \\
d \sigma / d \Omega \\
\left(\mathrm{cm}^{2} / \mathrm{sr}\right)\end{array}$ \\
\hline 1.0 & $(2.6 \pm 1.3) \times 10^{-11}$ & $(2.0 \pm 0.6) \times 10^{-11}$ & \\
\hline 1.6 & & & $(8.6 \pm 4.3) \times 10^{-12}$ \\
\hline 2.0 & $(6.0 \pm 1.4) \times 10^{-12}$ & $(5.2 \pm 0.7) \times 10^{-12}$ & $(2.6 \pm 0.6) \times 10^{-12}$ \\
\hline 2.8 & & & $(8.4 \pm 1.5) \times 10^{-13}$ \\
\hline 3.0 & $(2.9 \pm 0.6) \times 10^{-12}$ & $(2.0 \pm 0.3) \times 10^{-12}$ & \\
\hline 3.5 & & & $(4.8 \pm 0.9) \times 10^{-13}$ \\
\hline 4.0 & $(1.7 \pm 0.4) \times 10^{-12}$ & $(9.2 \pm 1.7) \times 10^{-13}$ & \\
\hline 4.8 & & & $(1.3 \pm 0.4) \times 10^{-13}$ \\
\hline 5.0 & $(8.3 \pm 1.7) \times 10^{-13}$ & $(5.6 \pm 1.1) \times 10^{-13}$ & \\
\hline 6.0 & $(4.7 \pm 1.1) \times 10^{-13}$ & & $(2.9 \pm 0.7) \times 10^{-14}$ \\
\hline 6.5 & & $(2.5 \pm 0.4) \times 10^{-13}$ & \\
\hline 7.2 & & & $(1.1 \pm 0.4) \times 10^{-14}$ \\
\hline 7.5 & $(2.9 \pm 0.9) \times 10^{-13}$ & & \\
\hline 8.0 & & $(1.2 \pm 0.3) \times 10^{-13}$ & \\
\hline 8.3 & & & $(4.3 \pm 1.5) \times 10^{-15}$ \\
\hline 9.0 & $(2.3 \pm 0.7) \times 10^{-13}$ & & \\
\hline 11.0 & $(9.7 \pm 2.1) \times 10^{-14}$ & $(5.6 \pm 1.5) \times 10^{-14}$ & \\
\hline 12.5 & $(7.8 \pm 2.2) \times 10^{-14}$ & & \\
\hline 13.5 & & $(1.7 \pm 0.7) \times 10^{-14}$ & \\
\hline 15.0 & $(4.8 \pm 2.1) \times 10^{-14}$ & & \\
\hline 16.0 & & $(9.3 \pm 2.6) \times 10^{-15}$ & \\
\hline 18.0 & $(2.0 \pm 0.5) \times 10^{-14}$ & $(3.8 \pm 1.0) \times 10^{-15}$ & \\
\hline 22.0 & $(1.4 \pm 0.3) \times 10^{-14}$ & & \\
\hline 25.0 & $(7.6 \pm 2.2) \times 10^{-15}$ & & \\
\hline
\end{tabular}


TABLE IV. Experimentally determined elastic angular differential cross sections for beryllium-ion-helium scattering in the center-of-mass frame for a beryllium ion with a laboratory energy of $56.25 \mathrm{keV}$.

\begin{tabular}{cc}
\hline $\begin{array}{c}\theta_{\text {c. m. }} \\
(\mathrm{mrad})\end{array}$ & $\begin{array}{c}56.25 \mathrm{keV} \\
d \sigma / d \Omega \\
\left(\mathrm{cm}^{2} / \mathrm{sr}\right)\end{array}$ \\
\hline 1.0 & $(1.4 \pm 0.2) \times 10^{-11}$ \\
1.5 & $(4.7 \pm 1.1) \times 10^{-12}$ \\
1.9 & $(2.2 \pm 0.8) \times 10^{-12}$ \\
2.3 & $(1.2 \pm 0.2) \times 10^{-12}$ \\
2.9 & $(8.1 \pm 1.7) \times 10^{-13}$ \\
3.4 & $(5.7 \pm 3.1) \times 10^{-13}$ \\
4.5 & $(2.5 \pm 1.9) \times 10^{-13}$ \\
5.5 & $(1.1 \pm 0.9) \times 10^{-13}$ \\
6.5 & $(5.7 \pm 5.3) \times 10^{-14}$ \\
\hline \hline
\end{tabular}

valid. The model calculations presented here obviously obey the simple scaling because the projectile ions are treated as a "heavy proton." This implies that the $43.75-\mathrm{keV} \mathrm{Li}^{+}+\mathrm{H}$ ADCS and the $143.75-\mathrm{keV} \mathrm{Na}^{+}+\mathrm{H}$ ADCS should be nearly identical. By comparing Figs. 1 and 2 it is obvious that the $\mathrm{Na}^{+}+\mathrm{H}$ ADCS follows the results of the model calculation whereas the $\mathrm{Li}^{+}+\mathrm{H}$ ADCS is falling off more rapidly at the larger scattering angles than is the model calculation. This is an indication that the simple scaling does not hold for these QOE collision systems. Over their common measured angular range $(6$ to $15 \mathrm{mrad})$ the ratio of the $\mathrm{Na}^{+}+\mathrm{H}$ ADCS to the $\mathrm{Li}^{+}+\mathrm{H}$ ADCS varies from about 3.5 to 4.8 whereas

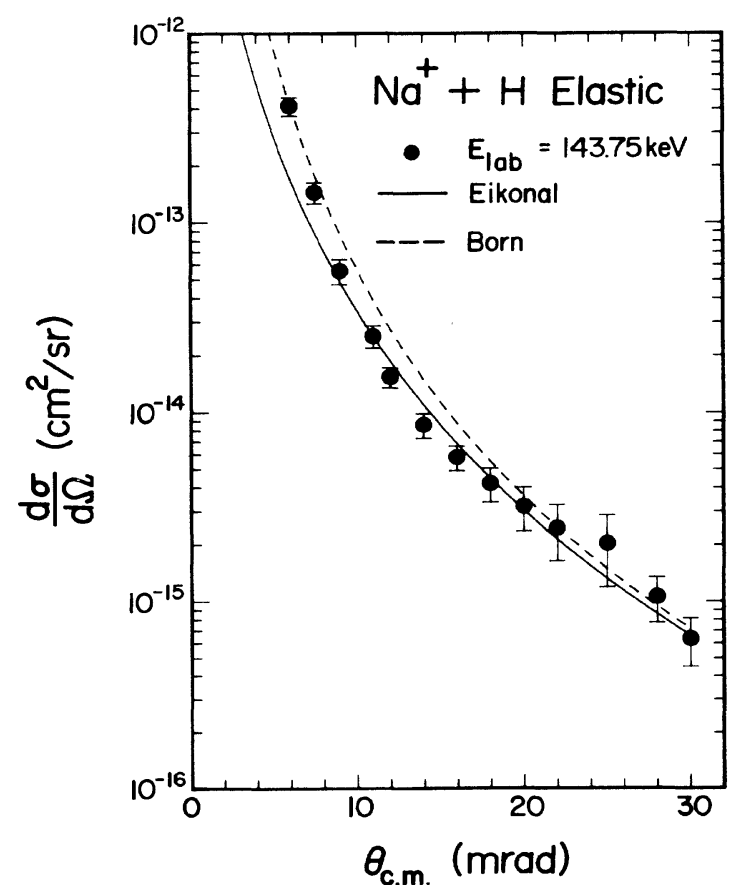

FIG. 1. Elastic angular differential cross section for sodiumion-hydrogen scattering in the center-of-mass frame for a sodium ion with a laboratory energy of $143.75 \mathrm{keV}\left(v=\frac{1}{2}\right.$ a.u.). Filled circle, present data; solid line, Eikonal calculation; dashed line, Born calculation.

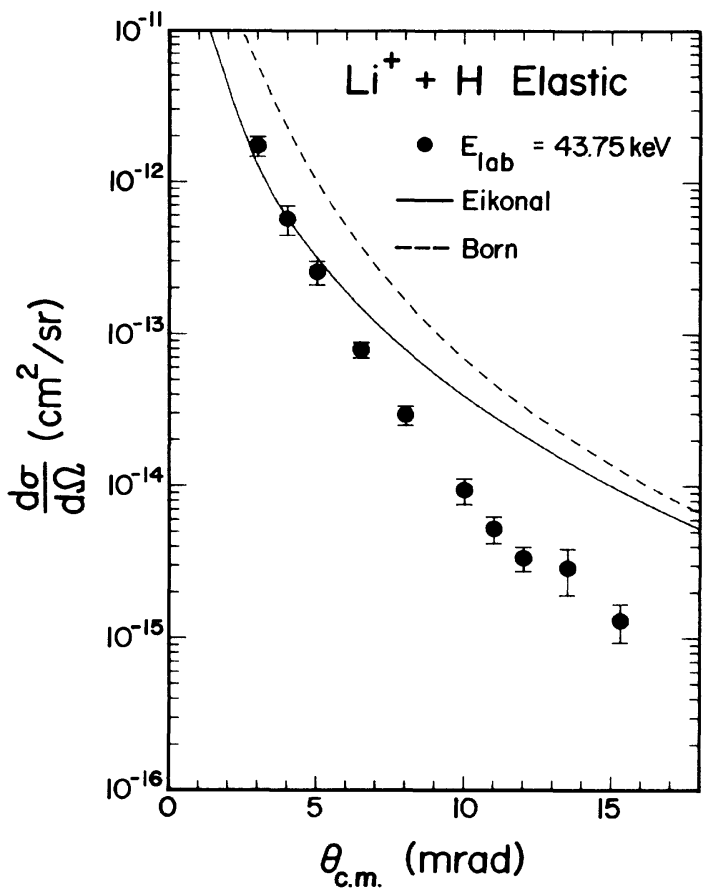

FIG. 2. Elastic angular differential cross section for lithiumion-hydrogen scattering in the center-of-mass frame for a lithium ion with a laboratory energy of $43.75 \mathrm{keV}\left(v=\frac{1}{2}\right.$ a.u. $)$. Filled circle, present data; solid line, Eikonal calculation; dashed line, Born calculation.

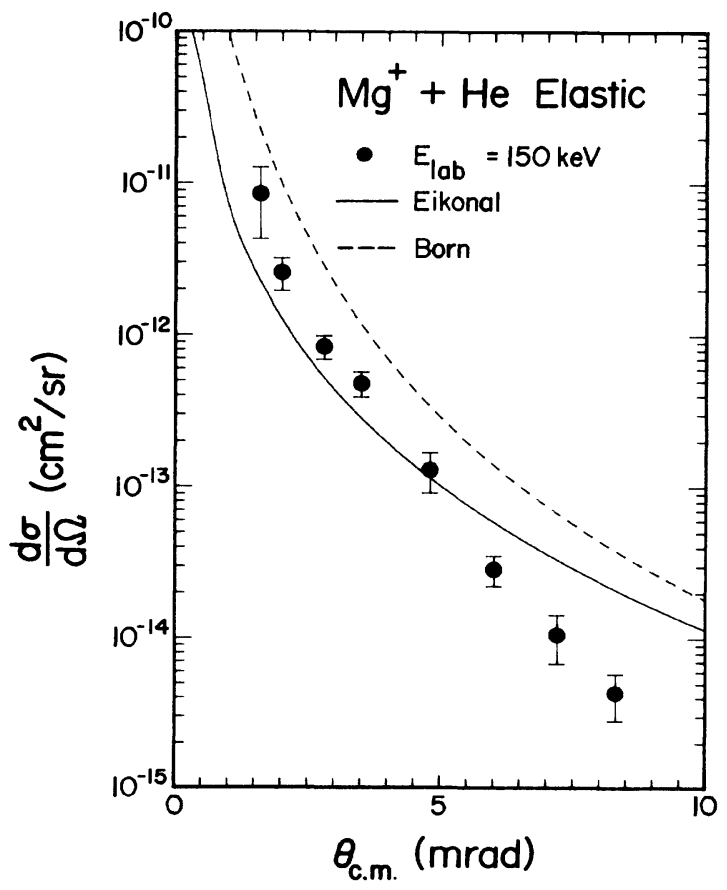

FIG. 3. Elastic angular differential cross section for magnesium-ion-helium scattering in the center-of-mass frame for a magnesium ion with a laboratory energy of $150 \mathrm{keV}\left(v=\frac{1}{2}\right.$ a.u.). Filled circle, present data; solid line, Eikonal calculation; dashed line, Born calculation. 
the ratio from the model calculation is about 0.85 for all angles in this range. Thus the experimental QOE results for a hydrogen target do not follow the simple scaling which was observed for different hydrogen-isotope projectiles.

We have plotted all our experimental data in the form of $\left(1 / \mu^{2}\right)(d \sigma / d \Omega)$ versus $\mu v \theta$. Plotted in this way the Born model calculation yields a universal curve for each target. Although the data all displayed a common trend when plotted in this manner, there was no simple scaling of the data. As mentioned above the $\mathrm{Li}^{+}+\mathrm{H}$ data decreased more rapidly than did the $\mathrm{Na}^{+}+\mathrm{H}$ data. The $\mathrm{Na}^{+}+\mathrm{H}$ data for different energies tended to separate as the momentum transfer increased. A similar trend occurred for the He target data.

Overall, the results of the model calculations are in fair agreement with the experimental data. Certainly the magnitude and general trend of the ADCS's are represented by this simple model. Our model assumes that the projectile nucleus is fully screened. At the larger scattering angles there is significant penetration of the electron cloud on the projectile ion by the target. Thus the target will interact with a larger effective charge because the projectile nucleus is no longer fully screened. This will lead to a larger ADCS at the larger scattering angles.

It is fortuitous that by ignoring the effect of penetrating the projectile electron cloud, the model approximations yield ADCS's that are similar in shape and have nearly the same magnitude as the experimental data. If the effect of charge-cloud penetration was taken into account, the results of the model calculations would be higher. Treating the projectile as a "heavy proton" is nearly compensating for the effect of the other inelastic scattering channels on the elastic scattering channel. The calculations do not explicitly take into account the effect of the other possible scattering channels on the elastic channel, especially the charge-transfer and ionization channels. These channels would remove flux from the elastic channel. This means the measured elastic ADCS is less than a simple perturbative calculation would predict if screening were included.

It would be necessary to carry out a multichannel calculation, which takes screening into account implicitly or through a pseudopotential, in order to be able to predict an accurate elastic ADCS. A multichannel calculation would presumably be able to predict an accurate elastic ADCS because it would be able to take into account the effect of the other scattering channels on the elastic scattering channel. However, a multichannel calculation for these collision systems at these intermediate energies is difficult. The ionization channel is particularly troublesome. We have observed this effect before in comparing our theoretical calculations and our experimental data for the $\mathrm{H}^{+}+\mathrm{He}$ elastic ADCS's. ${ }^{1}$ There is no projectile screening to consider in the $\mathrm{H}^{+}+\mathrm{He}$ collision system. The theoretical calculations yield an ADCS at the larger scattering angles that was significantly greater than the experimental data. The total differential cross section for the $\mathrm{H}^{+}+\mathrm{He}$ collision system was estimated in Ref. 1 by adding the measured elastic and charge transfer ADCS's to estimates of the total ionization and excitation ADCS's. The resulting total differential cross section was in good agreement with the calculated elastic ADCS. This is an illustration of how the other scattering channels can influence the elastic scattering channel.

Although the overall fair agreement of the results of our model calculation with the experimental data may be fortuitous, it is interesting to consider the general trends of the experimental data compared with the model calculation results. The QOE experimental results for a hydrogen target are all "steeper," i.e., more sharply peaked, than those of the model calculation. This effect is more pronounced for the $\mathrm{Li}^{+}+\mathrm{H}$ data than for the $\mathrm{Na}^{+}+\mathrm{H}$ data except at $63.89 \mathrm{keV}$. The best agreement between the model calculation and experimental results occurs at $143.75 \mathrm{keV}$ for the $\mathrm{Na}^{+}+\mathrm{H}$ collision.

The QOE experimental results for a helium target do not show a general trend as do the hydrogen target results. The model calculation and experimental results for the $\mathrm{Be}^{+}+\mathrm{He}$ collision system are in very good agreement. For the $\mathrm{Mg}^{+}+\mathrm{He}$ collision system the model calculation results are "steeper" than the experimental results at $30 \mathrm{keV}$. But at $150 \mathrm{keV}$ the experimental results are "steeper." The situation at $66.7 \mathrm{keV}$ is not as clear. The model calculation results are "steeper" at the smaller scattering angles below about $10 \mathrm{mrad}$ and the experimental results are "steeper" at the scattering angles.

In our previous QOE work concerning the excitation of the valence electron in the $\mathrm{Mg}^{+}+\mathrm{He}$ collision a significant divergence from the close-coupling calculations of Nielsen and Dahler ${ }^{8}$ occurred at center-of-mass angles of about 17 and $7 \mathrm{mrad}$ for the $66.7-$ and $150-\mathrm{keV}$ data, respectively. This divergence corresponded to an impact parameter of about 1.0 a.u. and is believed to be due to the onset of significant molecular excitation processes at the strongly avoided curve crossings near 1.0 a.u. ${ }^{22,23}$ Our elastic $\mathrm{Mg}^{+}+\mathrm{He}$ data at $150 \mathrm{keV}$ (see Fig. 3) shows a similar effect. Near $7 \mathrm{mrad}$ the data appears to start to fall off more rapidly. Unfortunately, our elastic $\mathrm{Mg}^{+}+$He data at $66.7 \mathrm{keV}$ ends at $18 \mathrm{mrad}$ so it is not clear whether or not the data is starting to fall off more rapidly after about $17 \mathrm{mrad}$. However, our elastic $\mathrm{Mg}^{+}+\mathrm{He}$ results at $150 \mathrm{keV}$ lend support to the interpretation of the previous results for the excitation of the valence electron in the $\mathrm{Mg}^{+}+\mathrm{He}$ system. If all of the significant scattering channels are not included in any multichannel calculation, the results for the elastic channel will be larger than they should be because the multichannel calculation conserves flux.

The results presented here indicate that modeling the projectile ion as a heavy structureless ion of charge $+1 e$ yields elastic angular differential cross sections that are in fair agreement with the experimental results at intermediate energies. More theoretical work is necessary in order to provide a more realistic theoretical description of elastic scattering at intermediate energies.

\section{ACKNOWLEDGMENT}

This research was supported in part by National Science Foundation Grant No. PHY-8406552. 
*Present address: Industrial Technology Department, Southwest Missouri State University, 901 S. National, Springfield, MO 65804.

'Present address: Insight Industries, 250 N. Court, Platteville, WI 53818

${ }^{1}$ J. L. Peacher, T. J. Kvale, E. Redd, P. J. Martin, D. M. Blankenship, E. Rille, V. C. Sutcliffe, and J. T. Park, Phys. Rev. A 26, 2476 (1982).

${ }^{2}$ E. Gerjuoy and B. K. Thomas, Rep. Prog. Phys. 37, 1345 (1974).

${ }^{3}$ K. Kobayashi and T. Ishihara, Phys. Rev. A 29, 3417 (1984).

${ }^{4}$ R. M. Potvliege, F. Furtado, and C. J. Joachain, J. Phys. B 20 1771 (1987).

${ }^{5}$ E. Rille, J. L. Peacher, E. Redd, T. J. Kvale, D. G. Seely, D. M. Blankenship, R. E. Olson, and J. T. Park, Phys. Rev. A 29, 521 (1984).

${ }^{6}$ N. Andersen and S. E. Nielsen, Adv. At. Mol. Phys. 18, 265 (1982).

${ }^{7}$ E. Redd, T. J. Gay, D. M. Blankenship, J. T. Park, J. L. Peacher, and D. G. Seely, Nucl. Instrum. Meth. B 24/25, 305 (1987).

${ }^{8}$ E. Redd, T. J. Gay, D. M. Blankenship, J. T. Park, J. L. Peacher, and D. G. Seely, Phys. Rev. A 36, 3475 (1987).

${ }^{9}$ T. J. Gay, E. Redd, D. M. Blankenship, J. T. Park, J. L. Peacher, and D. G. Seely, J. Phys. B 21, L467 (1988).

${ }^{10}$ J. T. Park, J. E. Aldag, J. M. George, and J. L. Peacher, Phys. Rev. A 14, 608 (1976)
${ }^{11}$ J. T. Park, J. M. George, J. L. Peacher, and J. E. Aldag, Phys. Rev. A 18, 48 (1978).

12J. T. Park, IEEE Trans. Nucl. Sci. NS-26, 1011 (1979).

${ }^{13}$ T. J. Kvale, D. G. Seely, D. M. Blankenship, E. Redd, T. J. Gay, M. Kimura, E. Rille, J. L. Peacher, and J. T. Park, Phys. Rev. A 32, 1369 (1985).

${ }^{14}$ J. T. Park, E. Redd, T. J. Kvale, and E. Rille, Rev. Sci. Instrum. 54, 1247 (1983).

${ }^{15}$ E. Redd, Ph.D. dissertation, University of Missouri-Rolla (1986).

${ }^{16}$ E. Redd and J. T. Park, Rev. Sci. Instrum. 55, 119 (1984).

${ }^{17}$ J. T. Park and F. D. Schowengerdt, Rev. Sci. Instrum. 40, 753 (1969).

${ }^{18}$ L. I. Schiff, Quantum Mechanics, 3rd ed. (McGraw-Hill, New York, 1968), see pp. 324 and 325 for the Born approximation and pp. 339-343 for the Eikonal approximation.

${ }^{19}$ H. L. Cox, Jr. and R. A. Bonham, J. Chem. Phys. 47, 2599 (1967).

${ }^{20}$ E. Clementi and C. Roetti, At. Data Nucl. Data Tables 14, 177 (1974); E. Clementi (unpublished).

${ }^{21}$ E. Rille, R. E. Olson, J. L. Peacher, D. M. Blankenship, T. J. Kvale, E. Redd, and J. T. Park, Phys. Rev. Lett. 49, 1819 (1982).

${ }^{22}$ C. Courbin-Gaussorgues, V. Sidis, and J. Vaaben, J. Phys. B 16, 2817 (1983).

${ }^{23}$ C. Courbin-Gaussorgues and V. Sidis, J. Phys. B 18, 699 (1985). 